\title{
On the Comparability of Cloud Fractions Derived from Whole Sky Imager and Ceilometer Data
}

\author{
Daniel Rodriguez
}

January 30, 1998

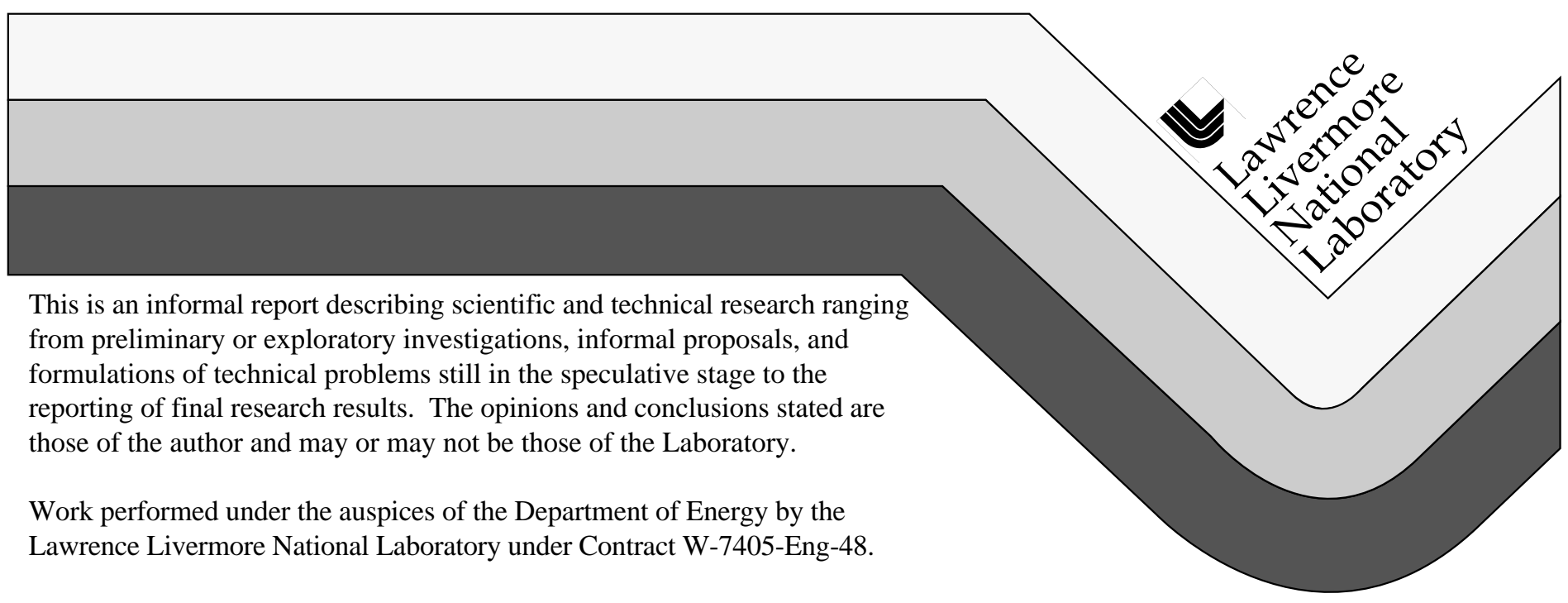




\section{DISCLAIMER}

This document was prepared as an account of work sponsored by an agency of the United States Government. Neither the United States Government nor the University of California nor any of their employees, makes any warranty, express or implied, or assumes any legal liability or responsibility for the accuracy, completeness, or usefulness of any information, apparatus, product, or process disclosed, or represents that its use would not infringe privately owned rights. Reference herein to any specific commercial product, process, or service by trade name, trademark, manufacturer, or otherwise, does not necessarily constitute or imply its endorsement, recommendation, or favoring by the United States Government or the University of California. The views and opinions of authors expressed herein do not necessarily state or reflect those of the United States Government or the University of California, and shall not be used for advertising or product endorsement purposes.

This report has been reproduced directly from the best available copy.

Available to DOE and DOE contractors from the Office of Scientific and Technical Information P.O. Box 62, Oak Ridge, TN 37831

Prices available from (423) 576-8401

Available to the public from the National Technical Information Service

U.S. Department of Commerce 5285 Port Royal Rd. Springfield, VA 22161 


\title{
ON THE COMPARABILITY OF CLOUD FRACTIONS DERIVED FROM WHOLE SKY IMAGER AND CEILOMETER DATA
}

\author{
Daniel Rodriguez
}

\section{INTRODUCTION}

The U.S. Department of Energy's Atmospheric Radiation Measurement (ARM) Program was established with the ultimate goal of improving the parameterizations of clouds and radiation being used in climate models (DOE, 1996). Achievement of this goal requires a better understanding of the processes that affect atmospheric radiation, with special attention given to cloud effects and their role in radiative feedback. To expand its basic knowledge, the ARM Program has fielded an extensive array of instruments...primarily surface-based, remote-sensing instruments....at its Clouds and Radiation Testbed (CART) site in north-central Oklahoma and south-central Kansas. The CART site, whose dimensions roughly correspond to those of a "typical" grid cell $(500 \times 500 \mathrm{~km})$ in a general circulation model, consists of a network of instrument clusters. These instruments are currently obtaining large volumes of detailed measurements. Many of them have been operating, almost continuously, for several years. These extended observations are beneficial to researchers for many reasons. For example, a greater variety of (climatologically relevant) cloud regimes can be sampled, instruments can be calibrated, or intercalibrated, more reliably, and data can be analyzed over extended periods to produce more robust and revealing statistics.

The ARM Program's most heavily instrumented site is its Central Facility, located near Lamont, OK. With respect to cloud observations, the instrumentation includes a whole sky imager, ceilometers, lidar, millimeter cloud radar, microwave profilers and radiosondes. Data from three of these instruments---the Whole Sky Imager (WSI), Belfort Laser Ceilometer (BLC) and Micropulse Lidar (MPL)---are used in this study, primarily to investigate the utility of using ceilometers, now strategically emplaced at four additional locations along the perimeter of the CART site, as aids in the characterization of fractional cloud cover across the entire domain. Such "grid-wide" characterizations could, for example, serve as inputs to Single Column Models for testing and refining cloud parameterizations. Climate modelers have long recognized that cloud fraction is probably the single most important cloud property for determining the surface and top-of-theatmosphere radiative fluxes (Arking, 1991). While a site-wide description of cloud fraction is an anticipated future goal, the major goal of this study is to investigate the comparability of time- and space-averaged cloud fractions at the Central Facility, using WSI data and a blend of BLC and MPL data.

\section{INSTRUMENTATION}

The Whole Sky Imager is a passive (i.e., non-emissive), automated system for measuring the sky radiance in approximately 0.3 degree increments over the entire sky dome (Shields et al., 1990). The sky radiances, uncalibrated at present, are acquired as two 16-bit images, which are used to determine the presence of clouds on a pixel-by-pixel basis. Acquisition of images under all possible lighting conditions (sunlight, moonlight and starlight) is aided 
by a rotating spectral filter wheel, containing neutral density, red $(650 \mathrm{~nm})$ and blue (450 $\mathrm{nm}$ ) filters. A solar/lunar occultor is positioned between the dome and the sun or moon to prevent direct sunlight or moonlight from producing artifacts in the image.

A WSI algorithm ratios the red and blue images to produce a ratio image. This image is compared, pixel-by-pixel, to a matching clear sky image, extracted from a library of background images that cover the full range of solar zenith angles. The algorithm identifies a pixel as being cloudy if the test image ratio exceeds the background ratio by a specified amount. The algorithm works quite well for opaque clouds. Thin clouds are more difficult to characterize because of difficulties in differentiating very thin cloud from fairly thick haze. While the WSI's solid-state CCD camera is capable of acquiring radiance images once every minute, a 10-minute retrieval is standard during routine operations.

The Micropulse Lidar is a zenith-pointing, optical remote sensing system designed primarily to determine the altitude of clouds overhead (Spinhirne et al., 1995). Its operating principle is the same as that of radars. It transmits pulses of energy and then measures the backscattered signal in $300 \mathrm{~m}$ range bins, up to about $20 \mathrm{~km}$. Analysis of the backscatter profile, obtained from 60 -s averages, with a threshold algorithm provides the lowest cloud base height in kilometers. The system, which has a narrow field of view (0.006 degrees), is highly sensitive; the MPL is able to detect both visible clouds and sub-visual cirrus.

Like the MPL, the Belfort Laser Ceilometer is a ground-based, optical remote sensing instrument. Clouds are detected by transmitting pulses of infrared light vertically into the atmosphere, and then measuring the scattered light from clouds and precipitation with a receiver telescope. The primary quantities measured by this system are the base heights of the lowest, second lowest and third lowest clouds, up to $7625 \mathrm{~m}$ over $7.6 \mathrm{~m}$ range gates. Its field of view is 0.17 degrees. While the data acquisition cycle can be user-selected, the current default rate is $30 \mathrm{~s}$.

\section{DATA ANALYSIS}

The ceilometer and sky imager data used in this study were collected at the Clouds and Radiation Testbed Central Facility between October 1 and December 31, 1996. This threemonth period corresponds to the first set of WSI data released by the ARM's Experiment Center (EC). While the EC continues to process and release WSI data, the study period is considered to be sufficiently long to reveal important trends.

Comparisons between the ceilometer and sky imager data are made by invoking the ergodic assumption, whereby ensemble, time and space averages are assumed to be equivalent. In regard to the ceilometer data, the applicability of the ergodic assumption depends mainly on the length of the time series under consideration and on the nature of the cloud field being sampled, i.e., the data must be sufficiently uncorrelated for the statistic being used to generate a useful result. No such assurance can be given for the hourly and daily comparisons in this study. Hourly values of cloud fraction from the WSI data are formed by averaging six cloud fraction estimates, based on an image frequency of ten minutes. A corresponding ceilometer estimate is formed by counting the number of cloud detections, and dividing the result by the number of measurements taken, typically 60, over the same hour. Among users of MPL and BLC data, it is generally acknowledged that the BLC is best at detecting clouds in the $0-3000 \mathrm{~m}$ range, while the MPL has greater 
sensitivity at higher altitudes (Turner, 1996). The practice of using one source of data or another, depending on the height range, is adhered to here. In this study, the 30-s BLC data has been temporally matched, within plus or minus $15 \mathrm{~s}$, to the 60-s MPL data. Normally, alternating BLC values are selected.

In reference to "daily averages", whole sky images are currently processed only for the daylight hours and only when the solar elevation angle is within plus or minus 80 degrees of zenith. Consequently, when the phrase "daily average" appears below, it actually means a daylight period lasting about eight hours. Algorithms for processing the remaining images are currently non-existent, but they will be developed in the near future. The procedures used in calculating the daily averages are analogous to the procedures used in forming hourly averages. The daytime WSI files contain hemispheric (horizon-to-horizon), sector (zenith angles between 0 and 80 degrees, with the resulting sky-dome fraction being divided into eight sectors), and ten-degrees-around-zenith (hereafter, referred to as 10degree) estimates of the cloud fraction. The hemispheric results were largely disregarded in this study, based on justifiable cause (see below). After applying the appropriate areal weights to the sector estimates of cloud fraction, the 10-degree and sector-weighted spatial averages were compared against the merged BLC and MPL temporal averages over hourly and daily periods. The results were further sub-divided, based on the prevailing heights of the cloud bases. From a quantitative standpoint, the correspondence between the 10-degree and ceilometer data was only marginally better than that offered by the sector-weighted data. Consequently, for reasons of "compactness" and with few exceptions, only those comparisons involving the 10-degree and ceilometer data will be presented here. In essence, biases in the daily averages can, rather easily, be inferred from the biases appearing in the hourly averages.

\section{RESULTS}

Figure 1 shows hourly-averaged, hemispheric cloud fractions plotted against their sectorweighted counterparts. The result reveals a distinct linear bias, with deviation from perfect agreement, indicated by the dotted line, increasing with increasing cloudiness. Cloudy sky images, like the one in Figure 2 for December 4, 1996 at 1535 UTC, suggest that all cloudy images have a "clear" annulus around their outer edge. The thickness of the ring changes from image to image, with the change ascribable to variations in the aerosol load and the cloud base height. In either event, the ring's relative (and artificial) contribution to the clear-sky component in the cloud fraction calculation increases with increasing cloud cover, a fact used here to justify the dismissal of hemispheric estimates from further consideration. 


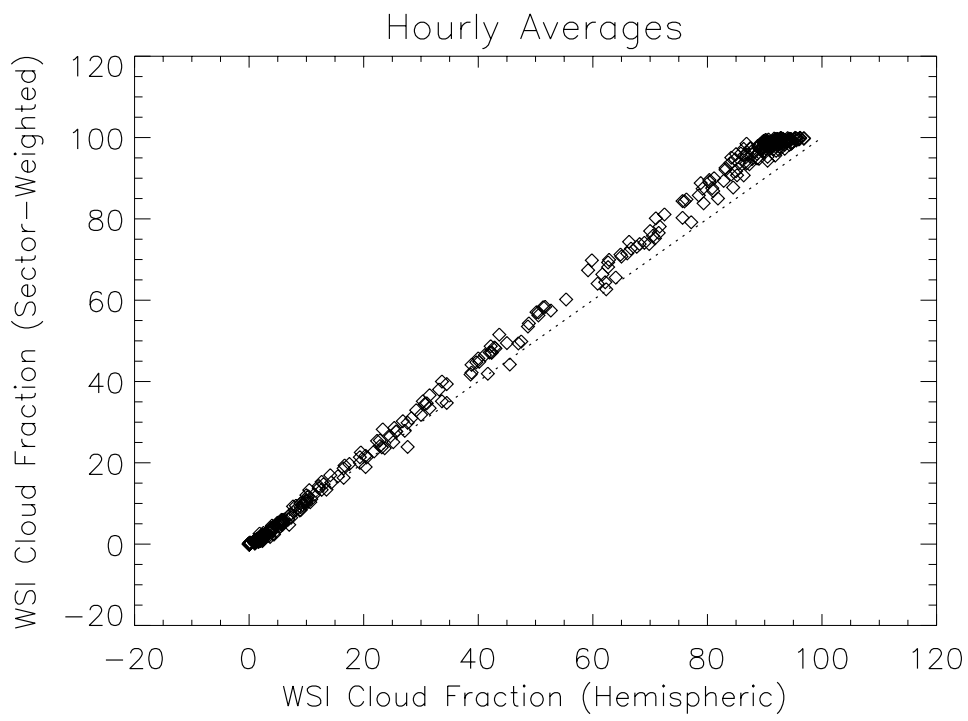

Figure 1. Comparison of hourly sector-weighted and hemispheric cloud fractions from WSI data.

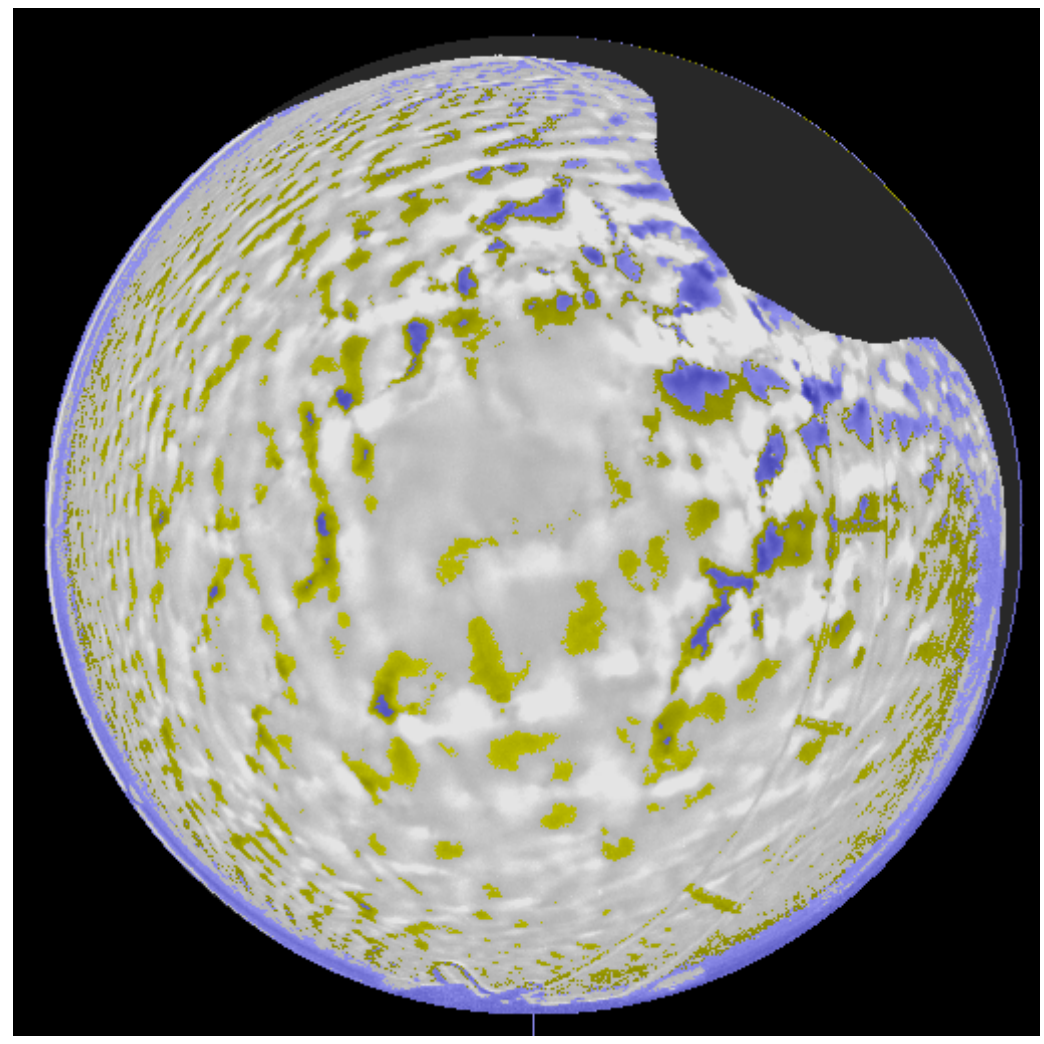

Figure 2. Processed whole sky image for 4 December 1996 at 1535 UTC.

Figure 3, which shows hourly 10-degree versus sector-weighted cloud fractions, reveals several interesting, but not entirely unexpected, features. The point concentrations in the lower-left and upper-right sectors indicate that the SGP weather was dominated by clear ( $0 \%$ time-averaged cloud fraction) or overcast (100\% time-averaged cloud fraction) skies during the Fall of 1996. The broadening of the distribution, corresponding to broken and 
scattered cloud fields, suggests that the ergodic assumption is poorly satisfied over hourly time scales. However, the symmetry of the distribution (the mean and standard deviation of the differenced pairs are $1 \%$ and $6 \%$, respectively) also suggests that the levels of agreement between the 10-degree and ceilomenter data, and between the sector-weighted and ceilometer data, will be comparable in an ensemble sense. This, in fact, has been borne out, as will be shown in the analysis below. The distribution in Figure 3 is most likely a reflection of the upper limit to which one can aspire in terms of time- and space-averaged data correlations since the 10-degree and sector-weighted results are derived from the same population of pixel values.

Figure 4 serves as an "early warning" that the correlation between the WSI and ceilometer cloud fractions will be significantly less than the correlation exhibited in Figure 3. The hourly 10-degree and merged BLC and MPL cloud fractions for October through December, 1996 are partitioned into $10 \%$ increments. The two sets of data that comprise these distributions are unpaired in time. For example, a $60 \%$ cloud fraction estimate from ceilometer data may, after time synchronization, correspond to a $0 \%$ cloud fraction estimate from WSI data. Both distributions indicate a preponderance of clear and overcast skies (within 10\%), but analyses of the WSI data indicates a greater incidence of clear skies, while the ceilometer data indicates a greater incidence of overcast skies.

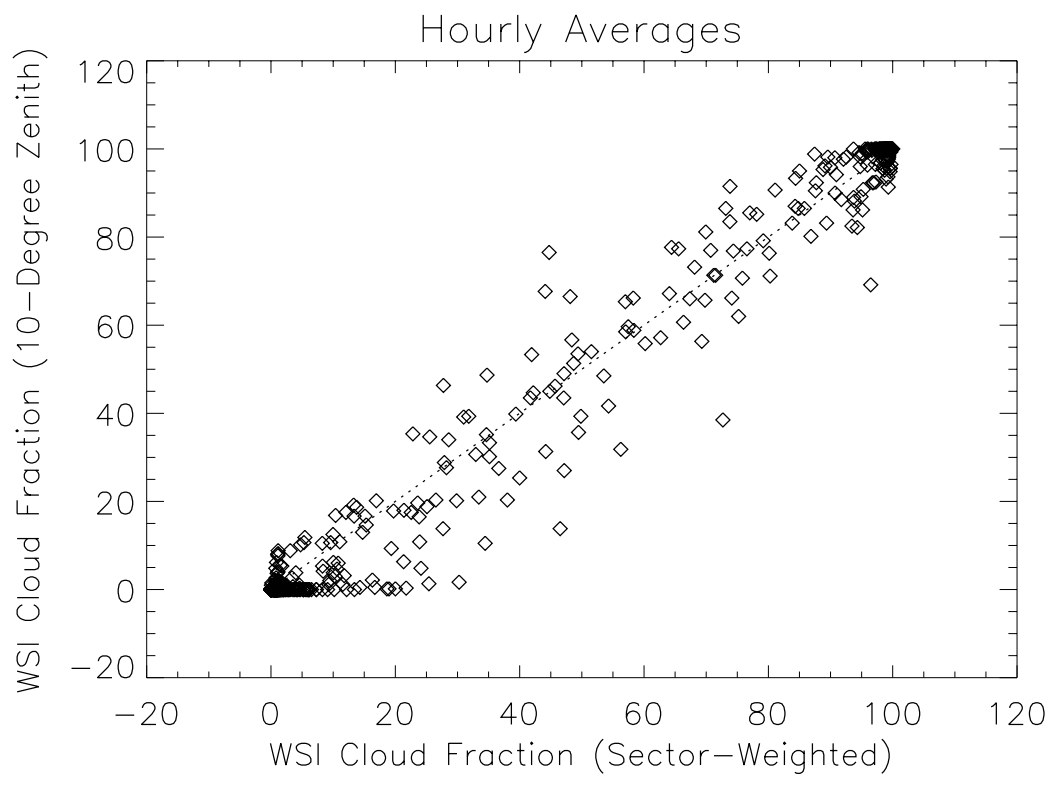

Figure 3. Comparison of hourly ten-degree and sector-weighted cloud fractions from WSI data. 


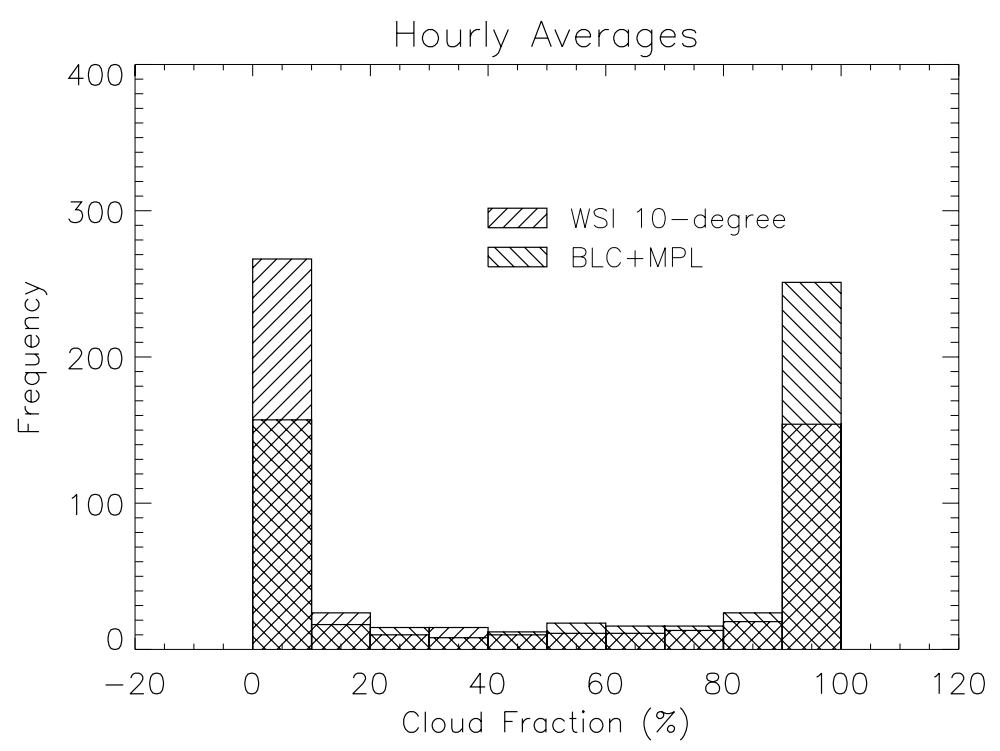

Figure 4. Frequency histogram of 10-degree WSI and merged ceilometer cloud amounts between 1 October 1996 and 31 December 1996 at the SGP CART site.

The scatter of points in Figure 5, based on temporal pairings of the WSI and ceilometer cloud fractions, reveals the true relationship between these data. In particular, the number of points that align themselves along the ordinate, which corresponds to clear (WSI) and varying cloudy (ceilometer) combinations, shows the poor correlation between these paired data. Inspection of many associated data records confirms that the WSI algorithm often produces cloud fraction estimates of zero, while the MPL, with its greater sensitivity, reports the presence of thin or sub-visual cirrus. As a case in point, the 10-degree cloud fraction estimate for the whole sky image in Figure 6 was zero, while the MPL reported an unbroken succession of cloud base heights at around $6720 \mathrm{~m}$ over 10 minutes, centered on the imaging time. In the current WSI algorithm, yellow patches within an image are equated with clear pixels, while white and grey areas correspond to cloudy pixels.

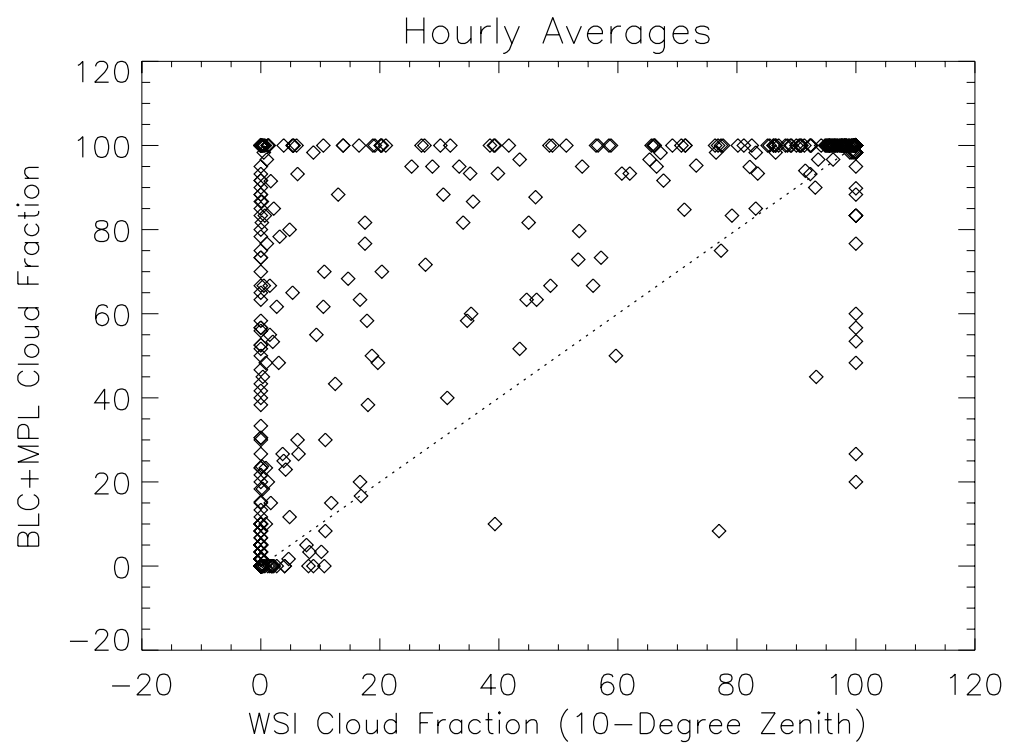


Figure 5. Scatter diagram of the hourly, paired-in-time cloud fractions from the 10-degree WSI and merged BLC and MPL data over the Fall months of 1996.

Although the points to the right of the diagonal line in Figure 5 constitute a small fraction of the 535 pairs of hourly values that were calculated and plotted, they merit attention because of the known, relative insensitivity of the WSI algorithm to cloud detection, as compared to the MPL and BLC algorithms. In particular, the outliers corresponding to combinations of WSI/ceilometer cloud fractions of approximately (100/20), (100/27) and (77/7) percent were unexpected. Before examining these cases, a review of how the BLC and MPL data are blended will make these outcomes more understandable. At a given time, a BLC-detected cloud is considered to be "valid" whenever its base height is greater than zero but less than or equal to $3000 \mathrm{~m}$ (a zero value indicates clear skies). Similarly, an MPL-detected cloud is considered to be valid whenever its base height exceeds $3000 \mathrm{~m}$. Cloud base heights, derived from the BLC and MPL measurements, that fall outside these respective ranges are ignored, unless one of the instruments is inoperative or its values extend beyond the normal range of acceptability. For these cases, all data from the remaining instrument are utilized without standard height restrictions. These rules are encapsulated in Table 1, which shows the various combinations that might exist between the BLC- and MPL-measured cloud base heights and the resulting cloud-field status.

With these rules in mind, an examination of the pairs of cloud fractions noted above revealed the following. In the first two instances, the MPL reported clouds 100 percent of the time over the hourly period, but their bases were all below $3000 \mathrm{~m}$. The BLC, on the other hand, reported clear skies 80 and $73 \%$ of the time; the remaining BLC measurements were out of range (see conditions 2 and 3 in Table 1). In these latter cases, MPL measurements were used, which explains the 20 and $27 \%$ estimates of cloud fraction from the ceilometers. The third and final case represents an oddity. The WSI, BLC and MPL all reported overcast conditions. However, most of the BLC-measured cloud base heights were slightly greater than $3000 \mathrm{~m}$ and most of the MPL-measured cloud base heights were slightly less than $3000 \mathrm{~m}$ (see condition 6 in Table 1). Using the stratagem adopted for combining the BLC and MPL data, the end result was a $7 \%$ estimate of cloud fraction, corresponding to those times at which the BLC and/or the MPL cloud bases were within acceptable height ranges. 


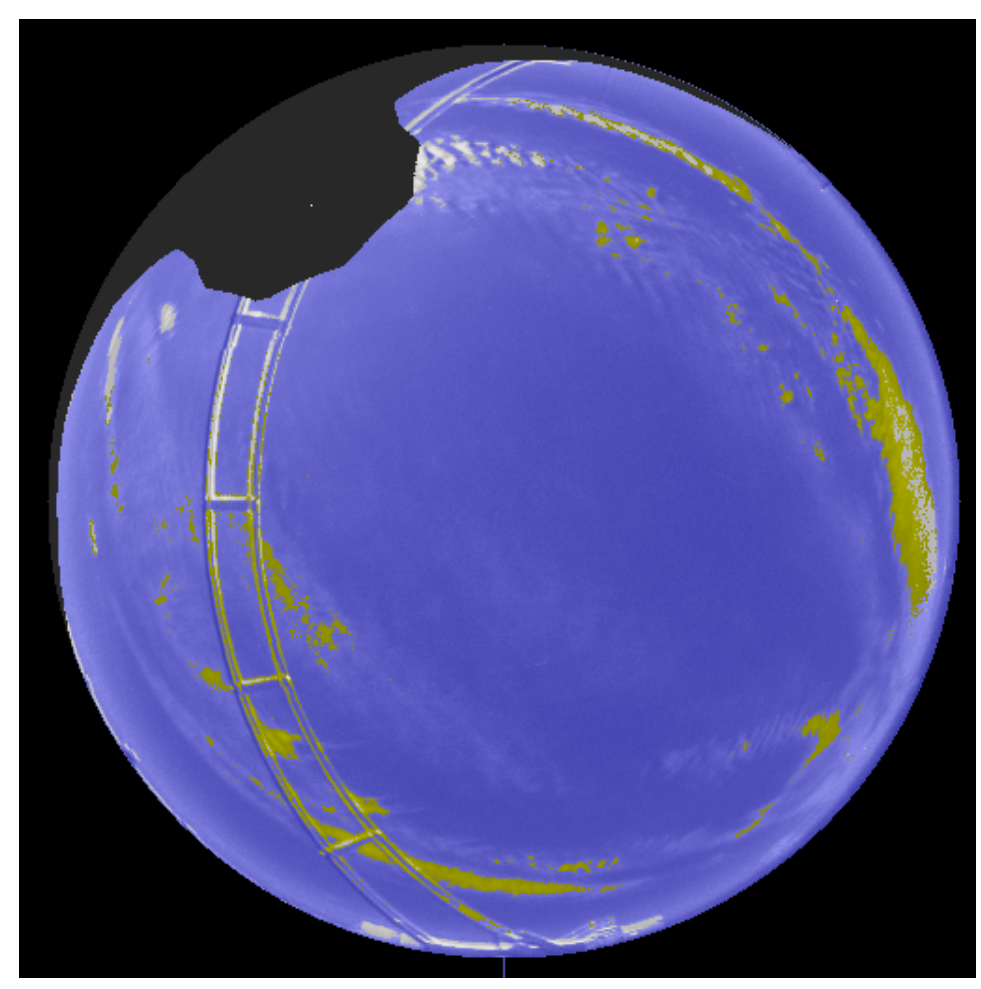

Figure 6. Processed whole sky image for 4 December 1996 at 2035 UTC.

Table 1. Acceptance rules for cloud detections based on combinations of BLC and MPL cloud base height measurements.

Cloud Base Height (m)

BLC

1.

2.

3.

4.

5.

6.

7.

8.

$<0$, or $>7350$
$<0$, or $>7350$
0
0
$1-3000$
$3001-7350$
$3001-7350$
$1-7350$

MPL

$\begin{array}{cl}0 & \text { clear } \\ 1-20000 & \text { cloudy } \\ <3000, \text { or }>20000 & \text { clear } \\ 3001-20000 & \text { cloudy } \\ 0-20000 & \text { cloudy } \\ 0-3000 & \text { clear } \\ 3001-20000 & \text { cloudy } \\ <0, \text { or }>20000 & \text { cloudy }\end{array}$

These outcomes prompted two additional lines of inquiry. First, the correspondence between the 1-min, paired-in-time BLC and MPL measurements was determined over the analysis period in the $0-5000 \mathrm{~m}$ height range. The resulting 22546 pairs of values fell within clear/clear, cloudy/cloudy and clear/cloudy categories 55, 38 and 7\% of the time, respectively. Second, given that the seven percent level of disagreement might well explain those points to right of the one-to-one correspondence line in Figure 5, the comparative WSI-ceilometer cloud fractions were recalculated, using only the MPL cloud detections and not a blend of MPL and BLC data. The results are shown in Figure 7. Although the 
distribution is perceptibly altered (cf., Figure 5), there is no quantitative improvement in the overall results, as determined by the tests described below.

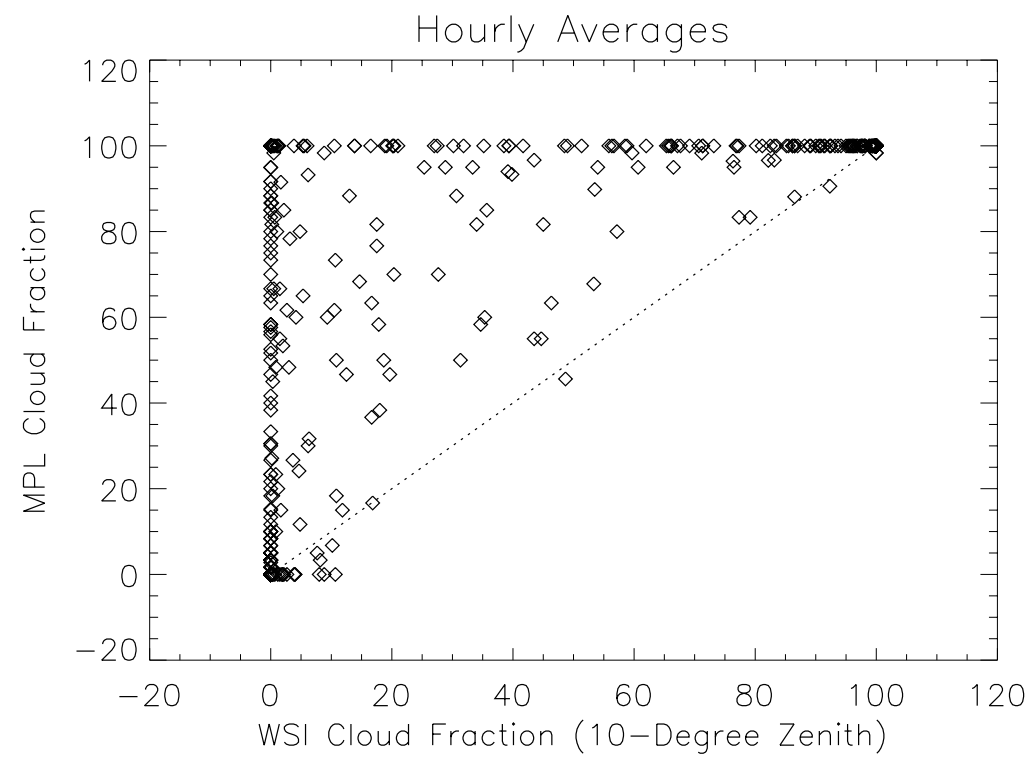

Figure 7. Scatter diagram of the hourly, paired-in-time cloud fractions from the 10-degree WSI and MPL data over the Fall months of 1996.

The point pairs in Figure 5 were binned into low $(<2000 \mathrm{~m})$, middle $(2000-5000 \mathrm{~m})$ and high $(>5000 \mathrm{~m}$ ) cloud ranges, according to the base heights reported by the ceilometers (see Figures 8-10). The principal reason for doing this was to isolate the effect of the MPL's greater sensitivity to cirrus (which might go unrecognized by the WSI algorithm depending on the cloud's opacity) on the distribution. However, it should be noted that this method of categorization is imperfect. During an averaging period, the clouds can, and often did, fall into different altitude ranges; for the sake of simplicity, the predominant (modal) range was allowed to prevail. Also, by categorizing the clouds strictly on the basis of cloud base height, the method fails to acknowledge variations in cloud depth. This factor may be important in broken or scattered cloud fields, i.e., the imaging algorithm would generally "see" both the bases and sides of the clouds, which would tend to bias the WSI result towards greater cloudiness, while the zenith-pointing ceilometers would see only the bases. Nevertheless, the end results of this procedure are illuminating. While the agreements in all three categories are considered poor, there is an obvious trend towards diminishing correspondence between the WSI- and ceilometer-derived cloud fractions with increasing cloud base height. For the most part, the skewness of the results shown in Figure 10 reflects the MPL's heightened sensitivity to cirrus, the detection of which often fell below the WSI's threshold. In contrast, the low clouds, represented by the distribution in Figure 8, generally had greater apparent optical thickness. In other words, the BLC and WSI were both capable of detecting their presence. 


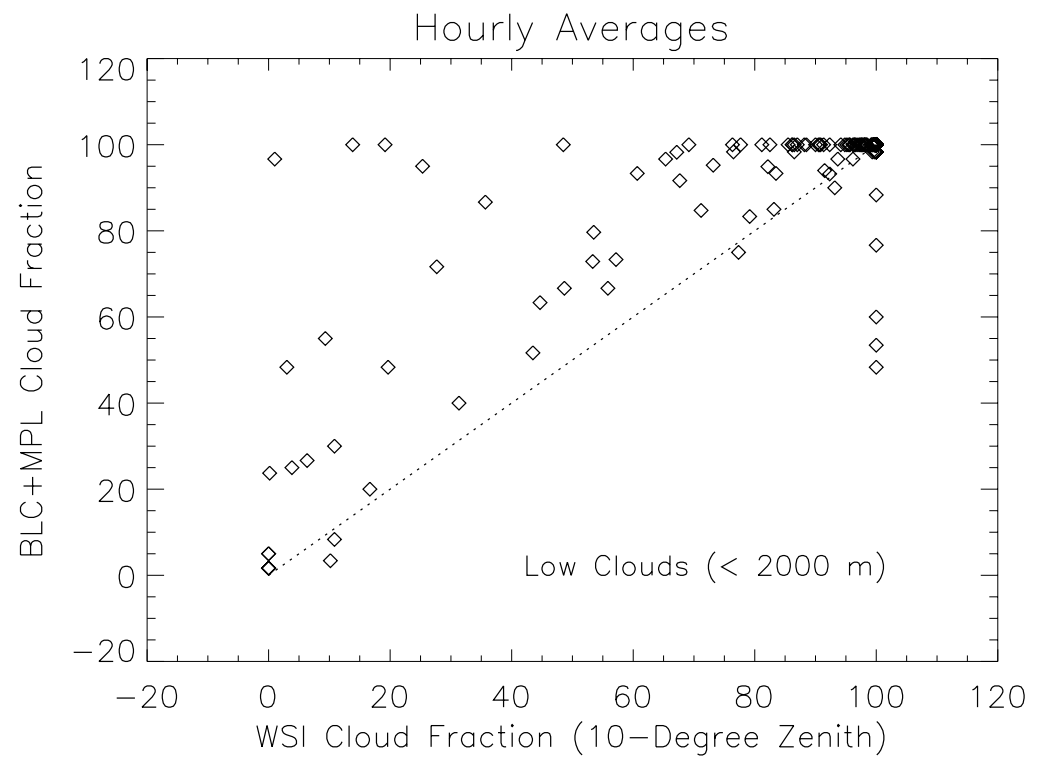

Figure 8. Hourly-averaged 10-degree and merged ceilometer cloud fractions for clouds whose base heights are below $2000 \mathrm{~m}$. 


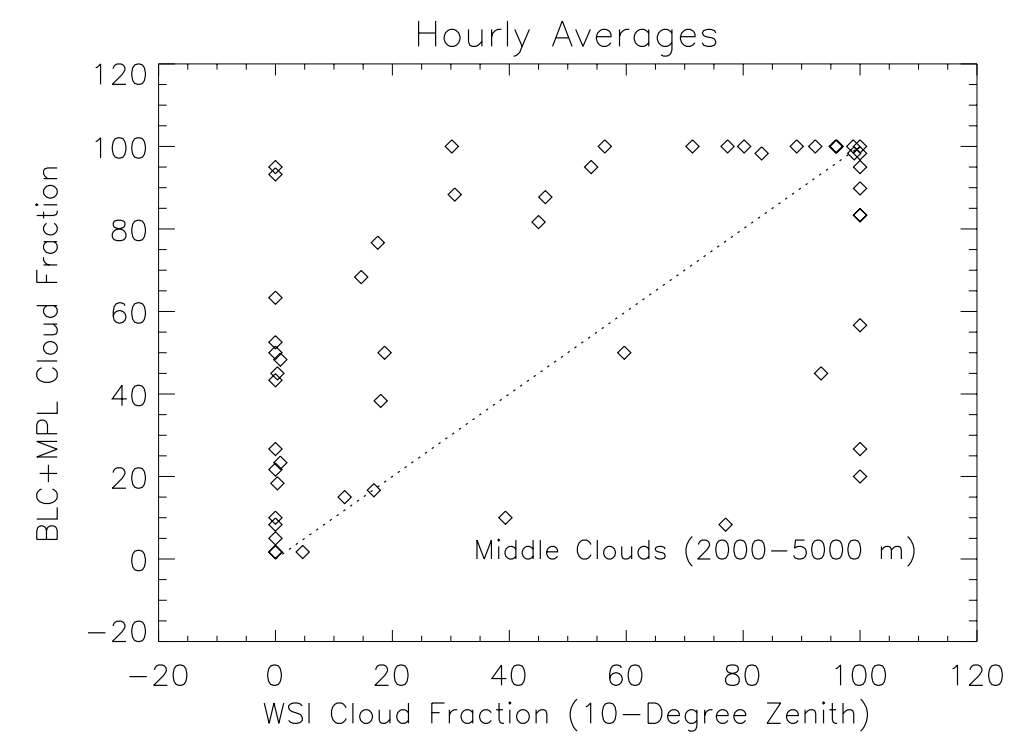

Figure 9. Same as Figure 8, except for clouds whose base heights are between 2000 and $5000 \mathrm{~m}$.

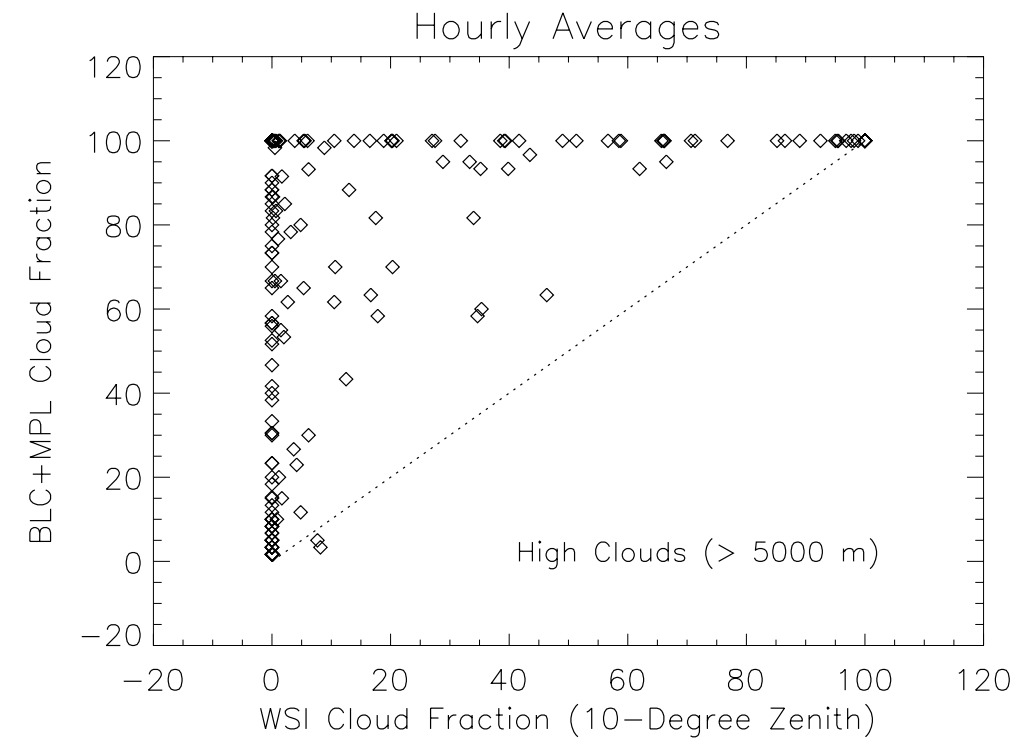

Figure 10. Same as Figure 8, except for clouds whose base heights are greater than $5000 \mathrm{~m}$. 
Obviously, treatment of the distributions shown in Figures 8-10 using standard statistics would be inappropriate because of their non-normality. However, there are non-parametric tests that can provide useful information. For example, the median of the differences of the ceilometer and 10-degree hourly cloud fractions, equal to $0.0 \%, 10.8 \%$ and $48.2 \%$ for the low, middle and high cloud results in Figures 8-10, is a useful quantitative measure, especially since it preserves information on distributional biases. (For purposes of comparison, the hourly sector-weighted versus ceilometer differences yielded medians of $1.0 \%, 12.6 \%$ and $48.8 \%$ in the low, middle and high cloud categories.) To demonstrate the effects of a longer averaging time, the daily cloud fraction results for the chosen ranges of cloud-base height are presented in Figures 11-13. Not surprisingly, the correspondence improves, but the biases seen in the hourly data are still observable in the daily data.

Finally, as a further demonstration that the correspondence between the hourly 10-degree WSI and ceilometer comparisons deteriorates as a function of range, the cumulative frequencies of the absolute differences of the ceilometer and 10-degree WSI cloud fractions in the three cloud categories are plotted in Figure 14. As expected, the best agreements occur for low cloud bases. Within this category, 35\% of the paired values agree exactly (note the tightly clustered points, corresponding to overcast and near-overcast conditions, in Figure 8 ), and $75 \%$ of the paired values agree within plus or minus $10 \%$. However, the correspondence decays with increasing cloud base height.

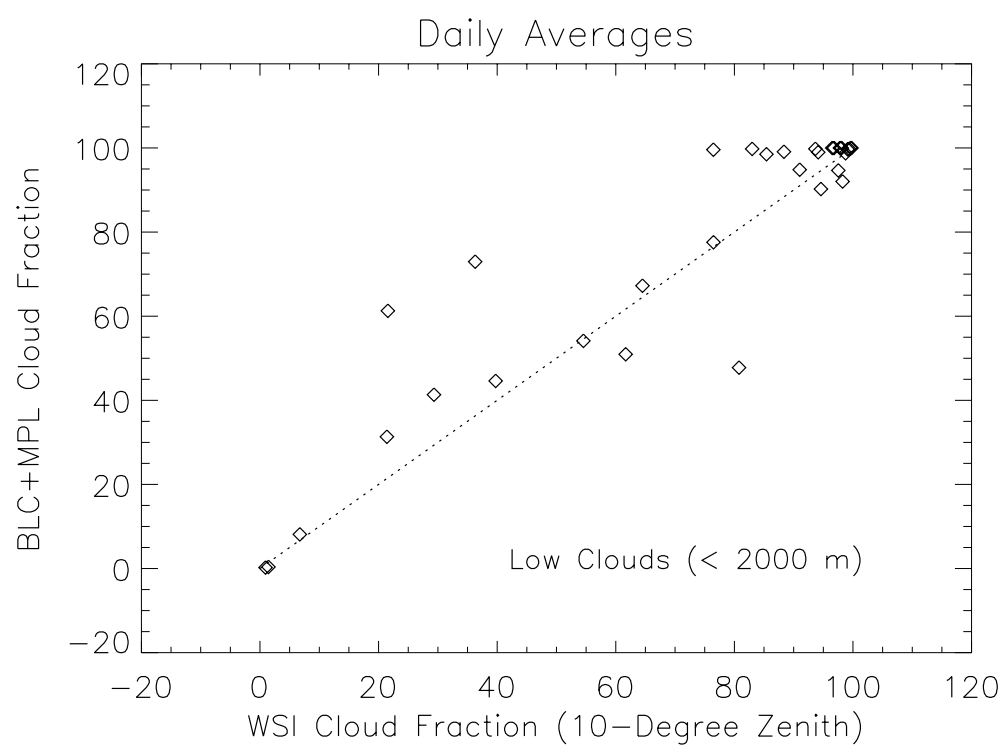

Figure 11. Daily-averaged 10-degree and merged ceilometer cloud fractions for clouds whose base heights are below $2000 \mathrm{~m}$. 


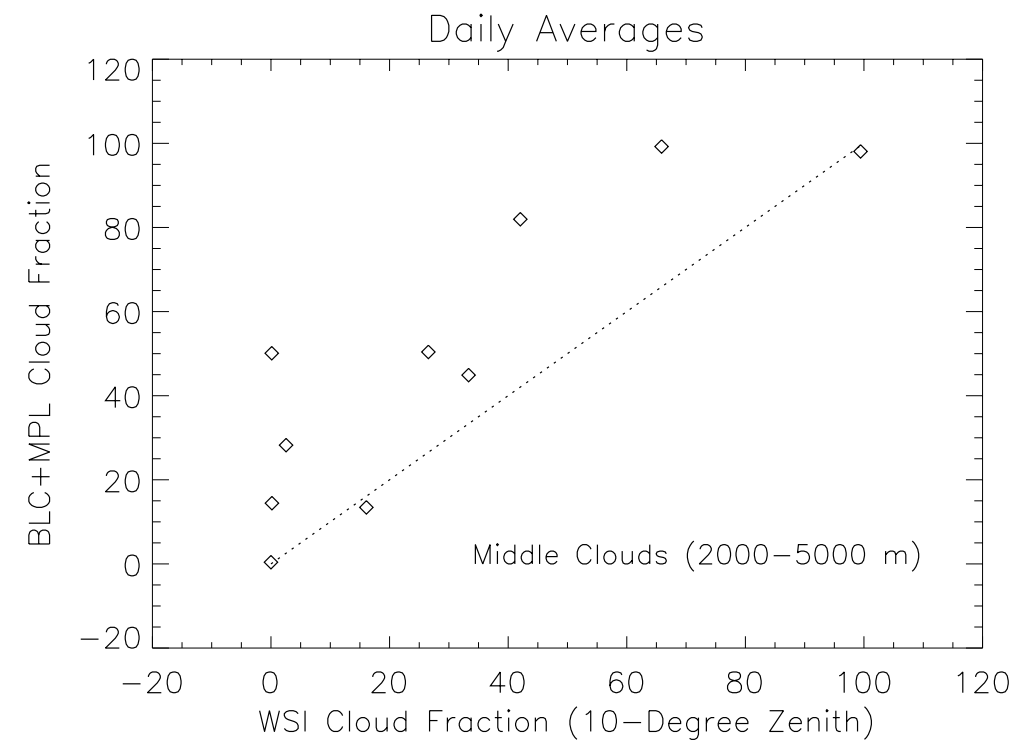

Figure 12. Same as Figure 11, except for clouds whose base heights are between 2000 and $5000 \mathrm{~m}$.

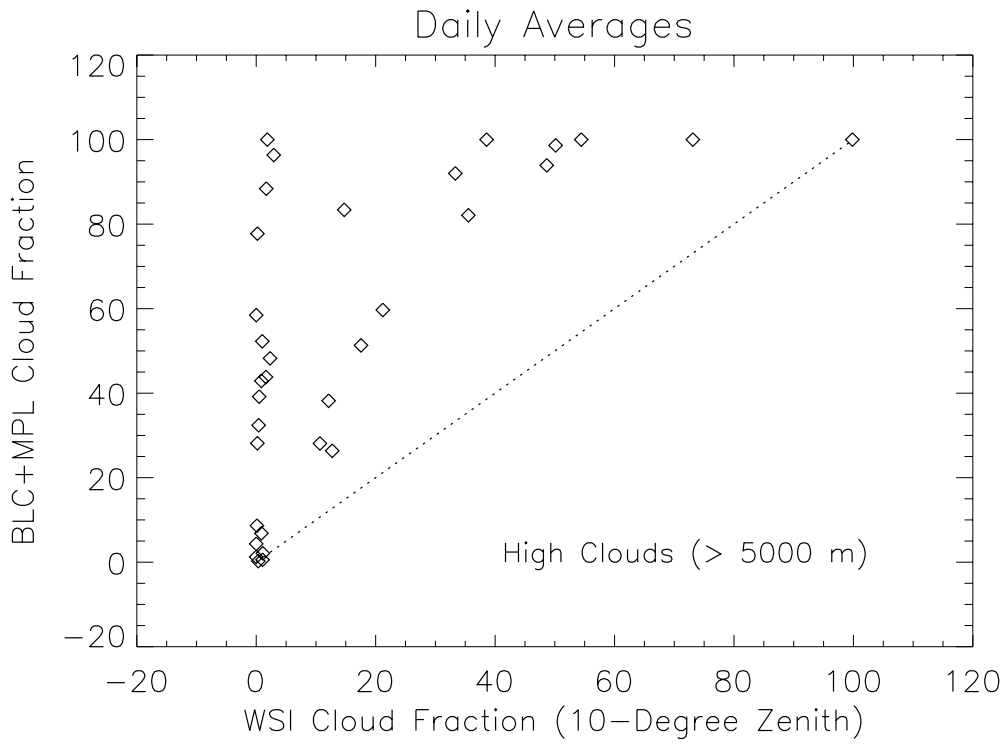

Figure 13. Same as Figure 11, except for clouds whose base heights are greater than $5000 \mathrm{~m}$. 


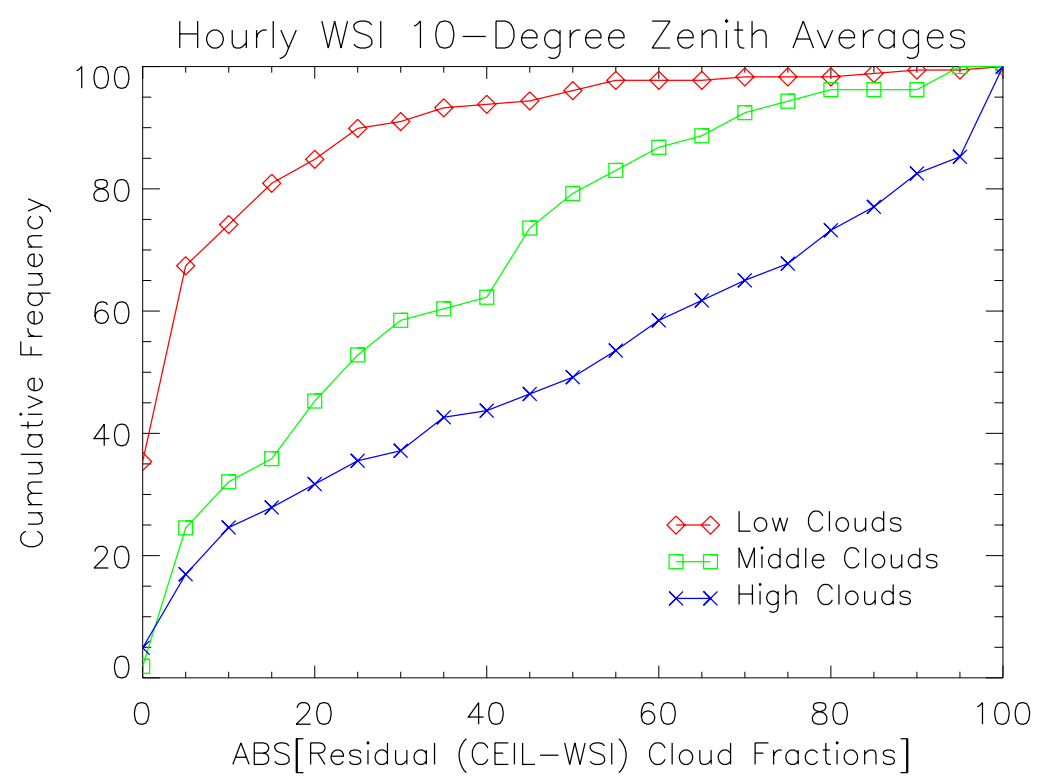

Figure 14. Cumulative frequencies of the absolute differences of the blended ceilometer and 10-degree WSI cloud fractions for cloud base heights in the low-, middle- and high-cloud categories.

\section{DISCUSSION AND RECOMMENDATIONS}

The recent release of Whole Sky Imager data from the ARM's Experiment Center has provided an opportunity to investigate the comparability of time- and space-averaged cloud fractions, using WSI data and a blend of Belfort Laser Ceilometer and Micropulse Lidar data. In principal, whole sky images (and estimations of cloud amount within these images) can serve as interpretive aids in analyzing the utility of time series of cloud detections from zenith-pointing ceilometers, used as estimators of cloud fraction. In reality, all three systems have their strengths and limitations, which must be clearly understood before their data can be combined to provide unambiguous determinations of fractional cloud cover.

There may be ways to improve the conformity of these cloud fraction estimates. Recommendations, accompanied by discussion, are given below.

1) Define the meaning of cloud more exactly.

The most basic question to be addressed is the definition of a cloud. Often, this definition varies, depending on the performance of a cloud-measuring instrument and its associated algorithms, or the definition is inexact. Lacking a universallyaccepted definition of a cloud, then the definition of cloud fraction becomes problematic. The ceilometers are affected by resolution problems, caused by their restricted fields of view and by the limited amounts of data used to estimate cloud fraction. However, the cloud-resolving capabilities of the ceilometers, especially the MPL, far exceed those of the WSI. The intrinsic value of the WSI, on the other hand, is that its algorithm provides these same estimates over the entire sky dome, and not just within a narrow band. The WSI algorithm is presently tuned to optically thick clouds because of historic concerns over the misinterpretation of 
haziness for cloudiness with the result that its sensitivity to the presence of cloud is less than that of the ceilometers.

2) Relate the significance of a cloud detection to cloud optical thickness.

Future investigations may reveal that, at times, the MPL is too sensitive for estimating fractional cloud cover. To use an exaggerated example, do two suspended water droplets 10 microns in diameter, placed $200 \mathrm{~m}$ apart, constitute a cloud (Di Girolamo and Davies, 1997). Perhaps, in regards to this study, a more meanful question might be, are all cloud detections of equal importance? For example, below a certain threshold, a cloud, whether detectable or not, will make an insignificant contribution to the surface energy budget. Consequently, the importance of a cloud detection can, perhaps, be related to its optical thickness. Recently, the ARM Experiment Center announced the release of several important data products, including optical depth, from a Langley analysis of Multifilter Rotating Shadowband Radiometer data. Unfortunately, this product is calculated only twice daily, thereby limiting its utility. However, other ARM-funded scientists are now attempting to couple range-allocated, particle size spectra with reflectivity profiles, using data from ARM's Millimeter Cloud Radar, which happens also to be situated at the Central Facility. This may, in time, yield estimates of cloud optical thickness, which could then be applied to the problem at hand.

3) Optimize the WSI cloud-identification algorithm.

This effort would include, among other things, the absolute calibration of WSI radiances, and the selection and application of a range of lower thresholds to data in which cirrus is the prevalent cloud form. In all likelihood, the results of this work will lead to the yellow-colored areas within a processed image being associated with cloud. However, it may be too much to hope that even a well-tuned algorithm could identify cloud within the 10-degree circle around zenith in Figure 6. Comparisons, like the ones conducted in this study, would help to identify the threshold value that produces optimal agreement between the WSI and ceilometer cloud fractions.

4) Consider alternatives to the current WSI algorithm.

The ARM instrument mentor for the Whole Sky Imager might consider the development of new, differential retrieval techniques, ones specifically geared to the detection of low (water) and high (ice) clouds, based perhaps on radiance measurements taken through an infrared filter in the Whole Sky Imager, or the development of an entirely new algorithm, premised on the recognition of clouds as fractal objects.

ARM-supported scientists tend to think in terms of data fusion, whereby data from complementary instruments can be synergistically combined to produce a new value-added product. In essence, it involves leveraging the strengths of instruments that possess one or more unique capabilities. As a pertinent example, it would clearly be advantageous to the ARM Program if a scheme was developed that rationally combined sky images with time series of cloud-base detections to generate cloud fractions as a function of height at the 
CART Central Facility. Thinking even further ahead, the marriage of satellite data with a spatially-distributed set of these estimates for purposes of characterizing the fractional cloud cover over the entire CART domain is certainly within the realm of possibility. 


\section{REFERENCES}

Arking, A., 1991: The Radiative Effects of Clouds and their Impact on Climate, Bull. Amer. Meteor. Soc., 71, 795-813.

Di Girolamo, L. and R. Davies, 1997: Cloud Fraction Errors Caused by Finite Resolution Measurements, J. Geophys. Res., 102, 1739-1756.

Shields, J.E., T.L. Koehler, M.E. Karr and R.W. Johnson, 1990: Automated Cloud Cover and Visibility Systems for Real Time Applications. University of California, San Diego, Scripps Institution of Oceanography, Marine Physical Laboratory, Optical Systems Group Technical Note No. 217.

Spinhirne, J.D., J.A.R. Rall, and V.S. Scott, 1995: Compact Eye Safe Lidar Systems, Rev. Laser Eng., 23, 112-118.

Turner, D.D., 1996: Comparisons of the Micropulse Lidar and the Belfort Laser Ceilometer at the Atmospheric Radiation Measurement Southern Great Plains Cloud and Radiation Testbed Site, Proceedings of the Sixth Atmospheric Radiation Measurement (ARM) Science Team Meeting, San Antonio, Texas, 4-7 March. U.S. Department of Energy, Washington, D.C.

U.S. Department of Energy (DOE), 1996: Science Plan for the Atmospheric Radiation Measurement Program (ARM), DOE/ER-0670T, U.S. Department of Energy, Washington, D.C. 


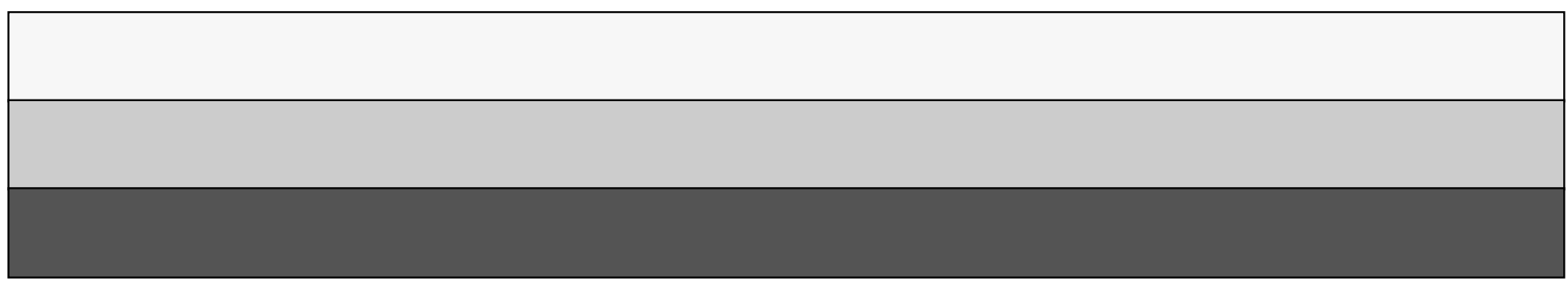

